\title{
Preclinical Models of Overwhelming Sepsis Implicate the Neural System that Encodes Contextual Fear Memory
}

\author{
Patricio T Huerta, ${ }^{1,2}$ Sergio Robbiati, ${ }^{1}$ Tomás S Huerta, ${ }^{1}$ Anchal Sabharwal, ${ }^{1}$ Roseann Berlin,${ }^{4}$ \\ Maya Frankfurt, ${ }^{3}$ and Bruce T Volpe,
}

${ }^{1}$ Laboratory of Immune and Neural Networks, The Feinstein Institute for Medical Research, Northwell Health, Manhasset, NY, United States of America; ${ }^{2}$ Department of Molecular Medicine, Hofstra Northwell School of Medicine, Hempstead, NY, United States of America; ${ }^{3}$ Department of Science Education, Hofstra Northwell School of Medicine, Hempstead, NY, United States of America; and ${ }^{4}$ Laboratory of Biomedical Science, The Feinstein Institute for Medical Research, Northwell Health, Manhasset, NY, United States of America

\begin{abstract}
Long-term sepsis survivors sustain cryptic brain injury that leads to cognitive impairment, emotional imbalance and increased disability burden. Suitable animal models of sepsis, such as cecal ligation and puncture (CLP), have permitted the analysis of abnormal brain circuits that underlie post-septic behavioral phenotypes. For instance, we have previously shown that CLP-exposed mice exhibit impaired spatial memory together with depleted dendritic arbors and decreased spines in the apical dendrites of pyramidal neurons in the CAl region of the hippocampus. Here we show that contextual fear conditioning, a form of associative memory for fear, is chronically disrupted in CLP mice when compared with sham-operated animals. We also find that excitatory neurons in the basolateral nucleus of the amygdala (BLA) and granule cells in the dentate gyrus (DG) display significantly fewer dendritic spines in the CLP group relative to the sham mice, although the dendritic arbors and gross morphology of the BLA and DG are comparable between the two groups. Moreover, the basal dendrites of CAl pyramidal neurons are unaffected in CLP mice. Taken together, our data indicate that structural damage in the amygdalar-hippocampal network represents the neural substrate for impaired contextual fear memory in long-term sepsis survivors. Further, our data suggest that brain injury caused by overwhelming sepsis alters the stability of the synaptic connections involved in associative fear. These results likely have implications for the emotional imbalance observed in human sepsis survivors.
\end{abstract}

Online address: http://www.molmed.org

doi: 10.2119/molmed.2015.00201

\section{INTRODUCTION}

Sepsis refers to the life-threatening shock and organ dysfunction caused by a dysregulated response to infection, which accounts for $\sim 750,000$ patients and $\sim 200,000$ deaths per year in the United States alone $(1,2)$. While mortality is highest during the first few months after onset, $50-80 \%$ of those patients who survive the acute stage and exit the hospital perish over the next two to eight years (3-6).
Additionally, long-term survival is accompanied by high morbidity, which is characterized by persistent cognitive impairment, emotional disturbances and increasing disability burden (7-9). It is therefore imperative to consider the brain as a crucial target organ for the chronic post-septic condition.

Recent preclinical studies using surgical and traumatic models of sepsis have shown that excessive activation

All authors contributed equally to this work.

Address correspondence to Bruce $T$ Volpe or Patricio T Huerta, The Feinstein Institute for Medical Research, 350 Community Drive, Manhasset, NY 11030, USA. Phone: 516-562-3384;

Fax: 516-562-2397; E-mail: bvolpe1@northwell.edu, phuerta@northwell.edu

Submitted September 8, 2015; Accepted for Publication November 2, 2016;

Published Online November 17, 2016.

พy⿱

Feinstein Institute

for Medical Research

Northwell Health

of the immune system during a septic episode, with resulting high levels of systemic cytokines, represents a critical causal factor in post-septic brain damage (10-26). Interestingly, there remains some controversy regarding the exact timing of the peak level for the different cytokines, as this ranges from hours to days after the septic insult (10-14,23). Since each sepsis paradigm has unique features, we focused on the cecal ligation and puncture (CLP) model because it replicates key aspects of human sepsis, such as $20-30 \%$ acute mortality, early hypotension and organ failure (27). CLP also mimics the most frequent form of polymicrobial gram-negative sepsis that occurs in hospitalized patients (28-33). The CLP model has been evaluated at the behavioral level, with studies showing that CLP survivors have transient ( 5-15 d) deficits in the open field test, the elevated plus maze and novel object 
recognition, as well as more lasting ( 30-60 d) deficits in inhibitory avoidance and forced swimming (34-40). Moreover, impaired contextual fear conditioning has emerged as a robust phenotype in post-septic animals $(21,41-45$, but see 46$)$. A study of CLP-surviving mice (at 1 month and 4 months post-surgery, compared with sham-operated mice) shows that they have sustained impairment in spatial memory but retain intact performance in other tasks (rotarod test, open field test and black-white alley) (10). Neuropathologic studies of these CLP mice reveal a progressive loss in the length and spine density of the apical dendrites of pyramidal cells from the CA1 region of the hippocampus (termed CA1 hereafter), which becomes apparent by $4 \mathrm{wks}$ post-surgery $(10,47)$. This delayed disruption of CA1 neurons, combined with their spatial impairment, indicates that the hippocampus is a crucial brain target for the long-term effects of sepsis. Notably, the hippocampus participates not only in encoding spatial cognition but also in encoding other domains, such as episodic memory and the contextual aspects of emotional memory. Therefore, we sought to extend our previous finding on impaired spatial memory (10) and examine whether CLP-treated mice also exhibit persistent abnormalities in contextual emotional memory.

Associative fear conditioning is a well-established approach to studying the neural basis of emotion (48-51). This behavioral paradigm represents an instance of Pavlovian classical conditioning, in which a conditioned stimulus (CS), typically a tone, is paired with an unconditioned stimulus (US), normally an electric shock that is delivered to the animal's footpad. Animals quickly learn to associate the CS with the US and thereafter express conditional responses, such as freezing (defined as absence of movement except for respiration), when they are presented with the CS alone, thus displaying associative fear memory. Studies have determined that the amygdala, and in particular the basolateral amygdala
(BLA), is essential for encoding this variant of the task (48-51). Interestingly, animals also learn to associate the context in which they receive the noxious stimulus (i.e., the context becomes a CS) and subsequently freeze when placed in the same environment in which they were shocked, thus showing contextual fear memory (48-51). It has been shown that the amygdala, together with CA1 and the dentate gyrus (DG), are essential for encoding this variant of fear memory. In this study, we find a significant disruption of contextual fear conditioning, together with altered dendritic architecture, in neurons within the BLA, CA1 and DG in the long-term CLP model.

\section{MATERIALS AND METHODS}

\section{Animals}

Experiments with male C57BL/6 mice (Charles River Laboratories) were performed in accordance with National Institutes of Health guidelines under protocols approved by the Institutional Animal Care and Use Committee of The Feinstein Institute for Medical Research, Northwell Health, Manhasset, New York. Animals were grouped in sets of five per cage, maintained on a reverse light schedule (dark period, 09:00 to 21:00) for the whole duration of the study (from their arrival from the vendor), with ad libitum access to food and water. They were 6-8 wks old at the time of CLP or sham surgery, after which they were analyzed behaviorally and anatomically, as shown in Figure 1A. One week before testing, mice were handled frequently for $5 \mathrm{~d}$ in 10-min sessions. Handling and behavioral testing occurred in the dark period of the circadian cycle.

\section{CLP Surgery}

Mice were anesthetized using ketamine (100 mg/kg) and xylazine $(8 \mathrm{mg} / \mathrm{kg})$ administered intramuscularly. A midline incision was made to expose the cecum. After ligation with a 4-0 silk ligature below the ileocecal valve, the cecum was punctured once with a 22-gauge needle and stool ( 1 $\mathrm{mm}$ ) was extruded from the hole. This CLP procedure is known to induce severe polymicrobial sepsis after a brief interval (52). The perforated cecum was placed back into the abdominal cavity, and the incision was closed with two layers of 6-0 Ethilon running sutures. All animals received normal saline resuscitation $(20 \mathrm{~mL} / \mathrm{kg}$ of body weight, injected subcutaneously) and a single dose of antibiotic (Primaxin, Merck, $0.5 \mathrm{mg} /$ mouse in $200 \mu \mathrm{L}$ sterile saline, injected subcutaneously), immediately after surgery. Sham-operated animals had the cecum isolated and then returned to the peritoneal cavity without being ligated or punctured, followed by antibiotic treatment and resuscitative fluid.

\section{Observational Screen}

Animals were monitored continuously for survival during the post-surgical period, as we expected some CLP-treated mice to perish shortly after surgery. The surviving animals were kept in groups of up to five individuals per cage and were assessed according to their cage number, which did not indicate the treatment. Moreover, the experimenter in charge of behavioral testing was not cognizant of the treatment, and therefore testing was performed in a blinded manner. Starting at d 30 postsurgery, mice were handled for $5 \mathrm{~d}$ in sessions of 5-10 min during the dark period of their circadian cycle. A behavioral screen, modified from the SHIRPA protocol $(10,53)$, was conducted to assess autonomic responses and neurological reflexes. The observational screen started with anatomical parameters (coat length, hair length and hair morphology), followed by observation in a cylindrical glass flask (height $15 \mathrm{~cm}$, diameter $11 \mathrm{~cm}$ ), which measured body position, spontaneous activity, respiratory rate, tremor occurrence, defecation and urination. Transfer to an arena $(55 \mathrm{~cm} \times 33 \mathrm{~cm})$ allowed for measuring of transfer arousal, latency to move in the arena and locomotion in the arena. This was continued with manipulations for measuring piloerection, 

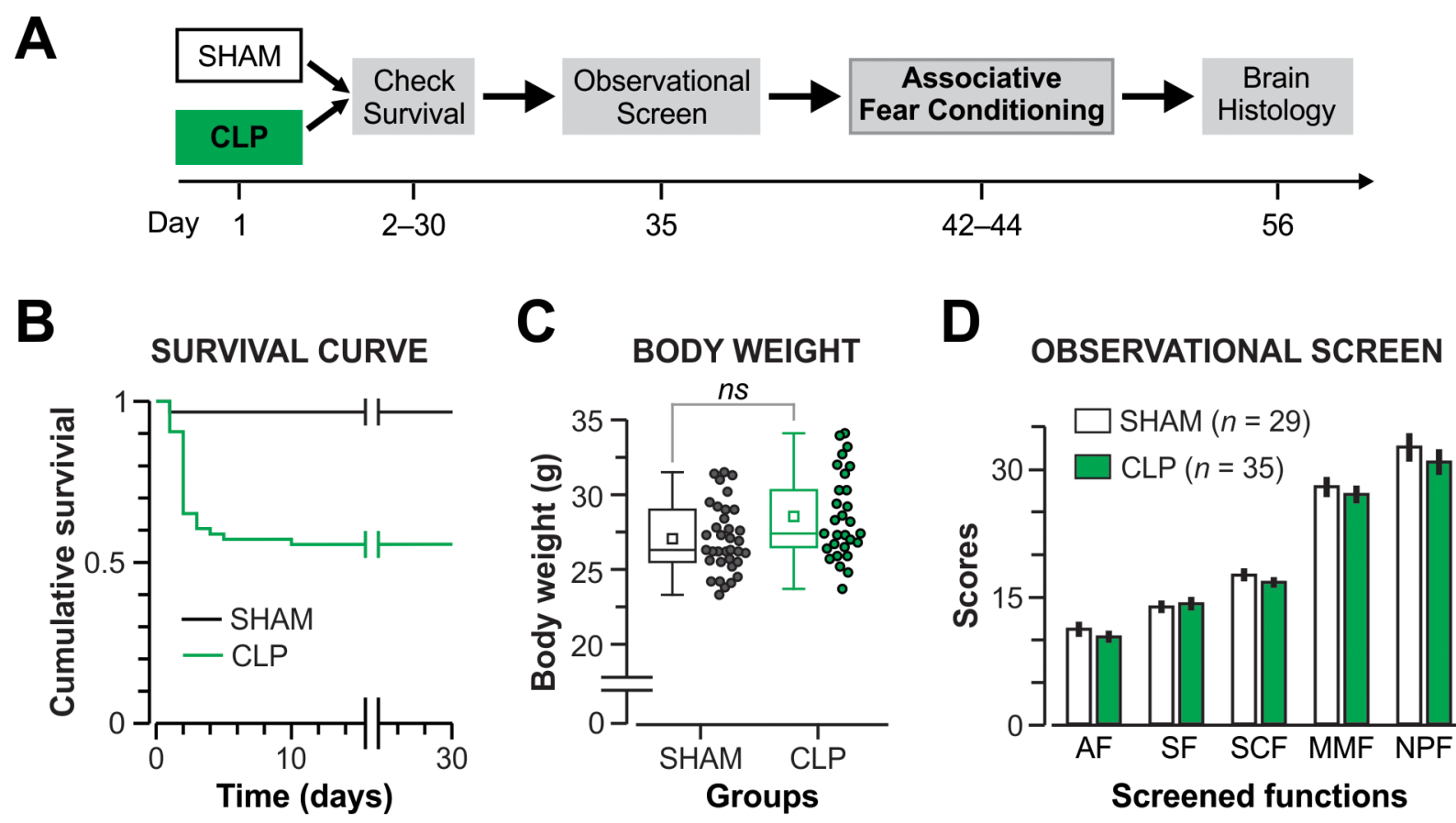

Figure 1. Design of the study. (A) Diagram of the timeline for the experiments in this study. Male C57BL/6 mice are subjected to cecal ligation and puncture (CLP) or sham surgery on $\mathrm{d}$ 1, which is followed by the indicated procedures. (B) Kaplan-Meier survival curve showing that some CLP-exposed mice perish within the first week post-surgery before the rest reach a stable survival rate of $56 \%$ by $d$ 11. (C) The box plot shows body weights at $35 \mathrm{~d}$ post-surgery, which are not significantly different between groups $(P=0.029, t=2.24, t$ test). (D) The graph describes the scores for the functions in the observational screen, as defined in Materials and Methods. All functions are comparable between CLP and sham groups ( $P>0.1$ for each function by t test). AF, autonomic function; SF, sensory function; SCF, spinocerebellar function; MMF, muscle and motoneuron function; NPF, neuropsychiatric function.

palpebral closure, startle response, gait, pelvic elevation, tail elevation, touch escape, positional passivity, trunk curl, limb grasping, visual placing, grip strength, body tone, pinna reflex, corneal reflex, toe pinch, body length, tail length, lacrimation, whisker morphology, provoked biting, salivation, heart rate, abdominal tone, skin color and limb tone. Measuring several reflexes (wire maneuver, righting reflex, contact righting, negative geotaxis) completed the screen. Throughout the screen, incidences of fear of the experimenter, irritability, aggressivity toward the experimenter, vocalizations and abnormal behavior were recorded. Finally, body weight was measured. The observed parameters were grouped according to five functional categories (53): muscle and motoneuron function, spinocerebellar function, sensory function, neuropsychiatric function and autonomic function. The summed scores for each function were averaged across mice belonging to the same group (CLP, sham) and these were then subjected to statistical analysis.

\section{Associative Fear Conditioning}

We have published these procedures (50), which are routinely used to study fear learning in rodents. Briefly, Chamber A was used for familiarization, CS-US training and the contextual memory test, and Chamber B was used for the tone memory test (Figure 2). Chamber A was a transparent Plexiglass shock box $(18 \mathrm{~cm}$ $\times 18 \mathrm{~cm} \times 30 \mathrm{~cm}$ ) equipped with a stainless steel grid floor (diameter of each grid $0.5 \mathrm{~cm}$, spacing $0.5 \mathrm{~cm}$; Precision-Regulated Animal Shocker, Coulbourn Instruments), which was dimly lit (orange bulb) and enclosed within a sound-attenuating environment. Chamber B was a cylindrical white Plexiglass chamber (diameter $20 \mathrm{~cm}$, height $30 \mathrm{~cm}$ ), which was brightly lit (white bulb) and contained bedding in the floor, similar to the home cage. A video camera was mounted at the top of the chambers to allow tracking of each animal. The software package FreezeFrame (Actimetrics) was used to deliver the CS-US protocols, track, and score freezing. The procedure occurred as follows (Figure 2): On the day before training (d 1), mice were familiarized to Chamber A with a 15-min session. On the day of training (d 2), they were placed in Chamber A, and after $3 \mathrm{~min}$ of acclimatization, were given five pairings of a tone CS (20 s, $5 \mathrm{kHz}, 80 \mathrm{~dB}$ ) that coterminated with a foot-shock US (1 s, $1 \mathrm{~mA})$, a paradigm known as delayed conditioning. The intertrial interval varied pseudorandomly between 90 and 120 s. On d 3, mice were first exposed to the contextual memory test in Chamber A; each mouse stayed in this apparatus for $10 \mathrm{~min}$ in the absence 

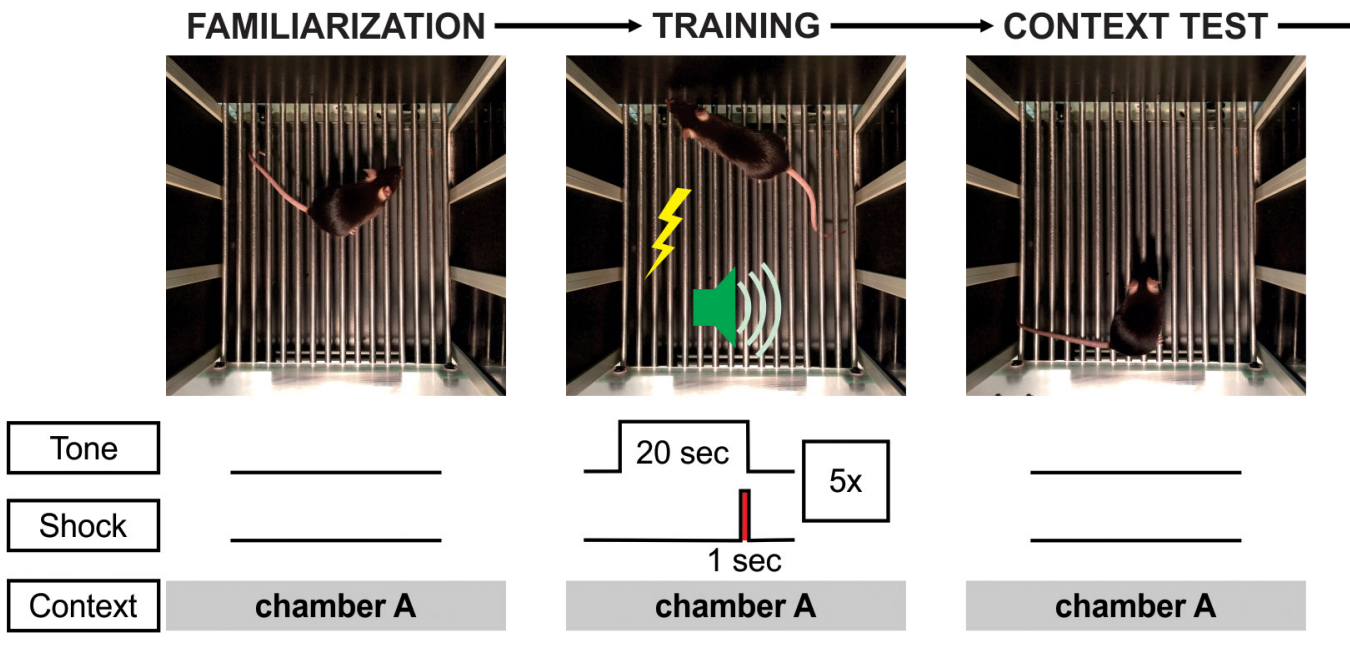
$\longrightarrow$ TONE TEST

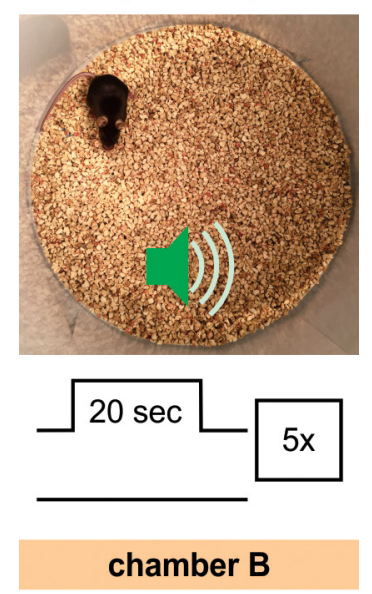

Figure 2. Protocol for associative fear conditioning. Male C57BL/6 mice are subjected to this 3 d paradigm at 6 wks post-surgery. For familiarization ( $d$ 1), each animal is exposed to Chamber A for $10 \mathrm{~min}$. For training ( $\mathrm{d}$ 2), the mouse is conditioned with five pairings of tone CS (20 s, $5 \mathrm{kHz}, 80 \mathrm{~dB}$ ) that coterminates with a foot-shock US ( $1 \mathrm{~s}, 1 \mathrm{~mA}$ ) in Chamber A. For the context test (d 3), the animal is placed in Chamber $A$ for $10 \mathrm{~min}$. For the tone test ( $\mathrm{d} 3$ ), the mouse is placed in Chamber $\mathrm{B}$, in which it received five tones (20 s, $5 \mathrm{kHz}$, $80 \mathrm{~dB}$ ).

of any foreground stimulus (such as the tone CS). At least $1 \mathrm{~h}$ after this test, mice underwent the tone memory test in Chamber B, in which, after a brief acclimation period, the mice received five test tones $(20 \mathrm{~s}, 5 \mathrm{kHz}, 80 \mathrm{~dB}$; variable interval 30-100 s). Data were expressed as percent of time spent freezing $(10 \mathrm{~s}$ blocks) and averaged across animals in each group.

\section{Brain Histology}

Golgi staining of neurons was performed with the FD Rapid Golgi Stain kit (FD Neuro Technologies) following our published protocols $(10,47,61)$. Briefly, $12 \mathrm{~d}$ after associative fear conditioning, we selected a subset of mice $(n=7$ per group) with average performance in the context test for their respective group for Golgi impregnation. Mice were euthanized with an injection of Euthasol $(100 \mu \mathrm{L})$, followed by rapid replacement of brain circulation with heparinized normal saline. Brains were removed and immersed in an impregnation solution (2 wks), cryoprotected (1 wk), cut on a cryostat (100 $\mu \mathrm{m}$ sections) and transferred to gelatin-coated slides. After drying, slides were stained and coverslipped. Using a Zeiss Axio imager microscope system, we identified sections containing the appropriate brain areas (BLA, CA1 and DG). Given that the section thickness and periodicity were similar in all cases, we sampled equivalent regions of interest across all experiments. On coronal sections (Bregma $-1.70 \mathrm{~mm}$ to $-2.40 \mathrm{~mm}$ ), regions of interest included the dorsal hippocampus and the BLA. For substructural sampling, we imaged neurons in the superior blade of the dentate gyrus immediately ventral to the sampled CA1; most often the BLA was also sampled on that coronal tissue section. We only used neurons in which the soma and dendrites were whole and unbroken in the projection field $(10,47)$. Tiled images of the neurons were acquired at $40 \times(Z$-stack step, $2.0 \mu \mathrm{m})$ and $100 \times$ oil (Z-stack step, $0.5 \mu \mathrm{m}$ ) with a Zeiss Axio imager. The images were transferred to an automated program for counting and analysis. For quantitation of the dendritic structures, a soma was identified and the entire length of the dendrite was traced using Neurolucida software (MBF). We used Sholl analysis to quantitate the length and complexity of the dendritic arbors. For spine quantitation, we traced dendrites that had been imaged with a $100 \times$ oil objective. We used Neurolucida to identify and count the spines present on the dendrite through the Z-stacked image. Brain tissue was also processed for standard vital stains by injecting animals with Euthasol (100 $\mu \mathrm{L})$, followed by rapid replacement of the brain circulation with heparinized saline, then $4 \%$ paraformaldehyde post-fixation for $1 \mathrm{~h}$, and infiltration with $30 \%$ sucrose overnight at $4^{\circ} \mathrm{C}$. Coronal brain slabs ( $5 \mathrm{~mm}$ thick) were isolated in a brain mold, mounted on a freezing stage and sectioned by microtome at $40 \mu \mathrm{m}$. Sections were collected in 0.1 M PB ( $\mathrm{pH} 7.4$ ). Every fourth section was mounted in $0.05 \mathrm{M}$ PB on gelatin-coated slides, air-dried and stained with cresyl violet.

\section{Statistical Analysis}

Data are presented as mean \pm standard error of the mean (SEM) or median ( $25 \%$ quartile, $75 \%$ quartile), as indicated. We used Student $t$ test and KolmogorovSmirnov test to examine statistical significance, which was defined as $P<0.05$. 


\section{RESULTS}

\section{Impaired Contextual Fear Memory in CLP-exposed Mice}

We sought to understand the lasting effects of a septic episode on emotional memory; therefore, we subjected male C57BL/ 6 mice to the CLP or sham surgery and examined their performance in associative fear conditioning at $6 \mathrm{wks}$ post-surgery (Figure 1A). Before this behavioral paradigm, we checked the animals' survival rate and found that at $30 \mathrm{~d}$ post-surgery, nearly all sham mice were alive (29 out of $30,97 \%$ ), whereas only $56 \%$ of the CLP-treated mice had survived (35 out of 63, Figure 1B). Moreover, at $35 \mathrm{~d}$ post-surgery, the two groups did not have differences in body weight (Figure 1C) and exhibited comparable behavioral performance in an observational screen $(10,53)$ that tested basic sensory, motor and autonomic functions as well as neurological reflexes (Figure 1D).

For fear conditioning (Figure 2), we studied CLP survivors $(n=35)$ compared with sham mice $(n=29)$ at $6 \mathrm{wks}$ post-surgery. We found that during the familiarization session, on $\mathrm{d} 1$, both groups behaved similarly without any sign of freezing (data not shown). On d 2, mice were exposed to five pairings of the tone CS that coterminated with the foot-shock US inside Chamber A, a paradigm known as delayed fear conditioning. Both groups showed increasing levels of freezing as training progressed (Figure 3A); by the end of the session, they displayed robust conditional response (\% freezing for the last $30 \mathrm{~s}$, mean \pm SEM: sham, $18.37 \pm 2.3 ;$ CLP, $17.26 \pm 1.82 ; P=0.7 ; t=0.38, \mathrm{t}$ test). On d 3, mice were subjected to the tone test, in which the tone CS alone was played five times in Chamber B; we found that both groups showed similar freezing levels during tone presentation (Figure 3B; sham, $16.89 \pm 3.29$; CLP, $22.31 \pm 3.18$; $P=0.24 ; t=1.18$, $\mathrm{t}$ test). Also, mice underwent the context test, in which they were placed in Chamber A for $10 \mathrm{~min}$; freezing levels were compared for the last $5 \mathrm{~min}$, revealing that the CLP group froze significantly less than the sham group during this session (Figure 3C; sham, $38.29 \pm 4.19$; $\mathrm{CLP}, 18.76 \pm 2.43 ; \mathrm{P}=8.71 \times 10^{-5} ; t=4.03$, $\mathrm{t}$ test), indicating that CLP mice had a deficit in contextual fear memory.

We sought to establish whether CLP survivors displayed a disruption in contextual fear memory at longer intervals after surgery. Therefore, we examined separate cohorts of mice at three time points (6, 8 and $\sim 22$ wks post-surgery). We performed associative fear conditioning in these animals (Figure 2) and found that the levels of freezing did not differ between the respective CLP and sham groups during the training phase or the tone memory test (data not shown). Conversely, we found that each CLP group was persistently impaired in the context memory test when compared with the corresponding sham group (Figure 4), demonstrating a permanent abnormality in contextual fear memory. Statistical analysis of the data (\% freezing, mean \pm SEM) using $t$ test was as follows: for the 41-44 d cohort: sham $(n=19), 38.33 \pm$ 5.92; CLP $(n=31), 20.46 \pm 2.56 ; P=0.01$; $t=2.77$; for the 50-65 d cohort: sham $(n=13), 38.99 \pm 4.14 ; \mathrm{CLP}(n=12), 20.23$ $\pm 4.32 ; P=0.0047 ; t=3.13$; for the 78-176 d cohort: sham $(n=21), 31.25 \pm 4.7 ;$ CLP $(n=12), 10.35 \pm 3.29 ; P=9.93 \times 10^{-4} ; t=3.64$.

\section{Altered Dendritic Spine Density of BLA and DG Neurons in CLP-exposed Mice}

The detailed structural analysis was prompted by the normal appearance of the histopathology with a Nissl stain (data not shown). Associative fear conditioning depends on the structural integrity of the amygdala (48-51); therefore, we analyzed Golgi-stained excitatory BLA neurons (Figure 5A) in terms of their dendritic arbors and density of dendritic spines. Mice from the CLP $(n=7)$ and sham $(n=7)$ groups underwent anatomical analysis $8 \mathrm{wks}$ after the surgical procedure (12 d after fear conditioning). We used Sholl analysis to quantify the complexity of dendritic arbors, which revealed that BLA neurons from the sham and CLP groups ( $n=73$ in each group, $\sim 10$ neurons per animal) had no significant differences in dendritic complexity (Figure 5B; $P=0.9, D=0.18$, Kolmogorov-Smirnov test) or length (Figure 5C-E). Remarkably, quantification of spine density within BLA dendrites (sham, $n=67$; CLP, $n=89$; Figure $5 \mathrm{~F}$ ) revealed highly significant differences between groups (Figure 5G; number of spines per $\mu \mathrm{m}$, mean $\pm \mathrm{SEM}$ : sham, $0.91 \pm 0.03$; CLP, $0.47 \pm 0.02$; $P=2.6 \times 10^{-20} ; t=11.3, \mathrm{t}$ test $)$. Given that spine density can be a somewhat variable parameter, we decided to analyze another set of mice (CLP, $n=3$; sham, $n=3$ ) that were prepared at a later stage (12 d after fear conditioning as before, but 24 wks after surgery) and indeed we found that the anatomical differences between groups remained statistically sound (Figure 5H; number of spines per $\mu \mathrm{m}$, mean \pm SEM: CLP, $0.34 \pm 0.01$; sham, $0.72 \pm 0.03 ; P=4 \times 10^{-16} ; t=11.1$, t test $)$.

Although fear memory is mediated by the amygdala, the DG also contributes to encoding contextual fear memory. Here we used mice from the CLP and sham groups ( $n=7$ each) to measure the dendritic branching and spine density of granule cells from the DG (Figure 6A), and determined by Sholl analysis (Figure 6B) that there was no difference in dendritic length between groups (Figures 6C-E), but quantification of synaptic spines showed significant differences between groups (Figures 6F-G; number of spines per $\mu \mathrm{m}$, mean $\pm \mathrm{SEM}$ : sham, $1.07 \pm 0.04 ;$ CLP, $0.88 \pm 0.06$; $P=0.01 ; t=2.5$, $t$ test $)$.

Additionally, we investigated the status of dendritic spines in the basal dendrites of CA1 cells (Figures 7A and B) and, interestingly, found a tendency for fewer spines in the CLP group that did not reach statistical significance (Figure 7C; number of spines per $\mu \mathrm{m}$, mean \pm SEM: sham, $0.94 \pm 0.04 ; C L P$, $0.84 \pm 0.03 ; P=0.05 ; t=1.9, \mathrm{t}$ test $)$. In previous studies of CLP mice, we reported that the apical dendrites of CA1 pyramidal cells had lower spine density (10) and significantly shorter dendritic length (47) when compared with sham apical dendrites (Figure 7D). 

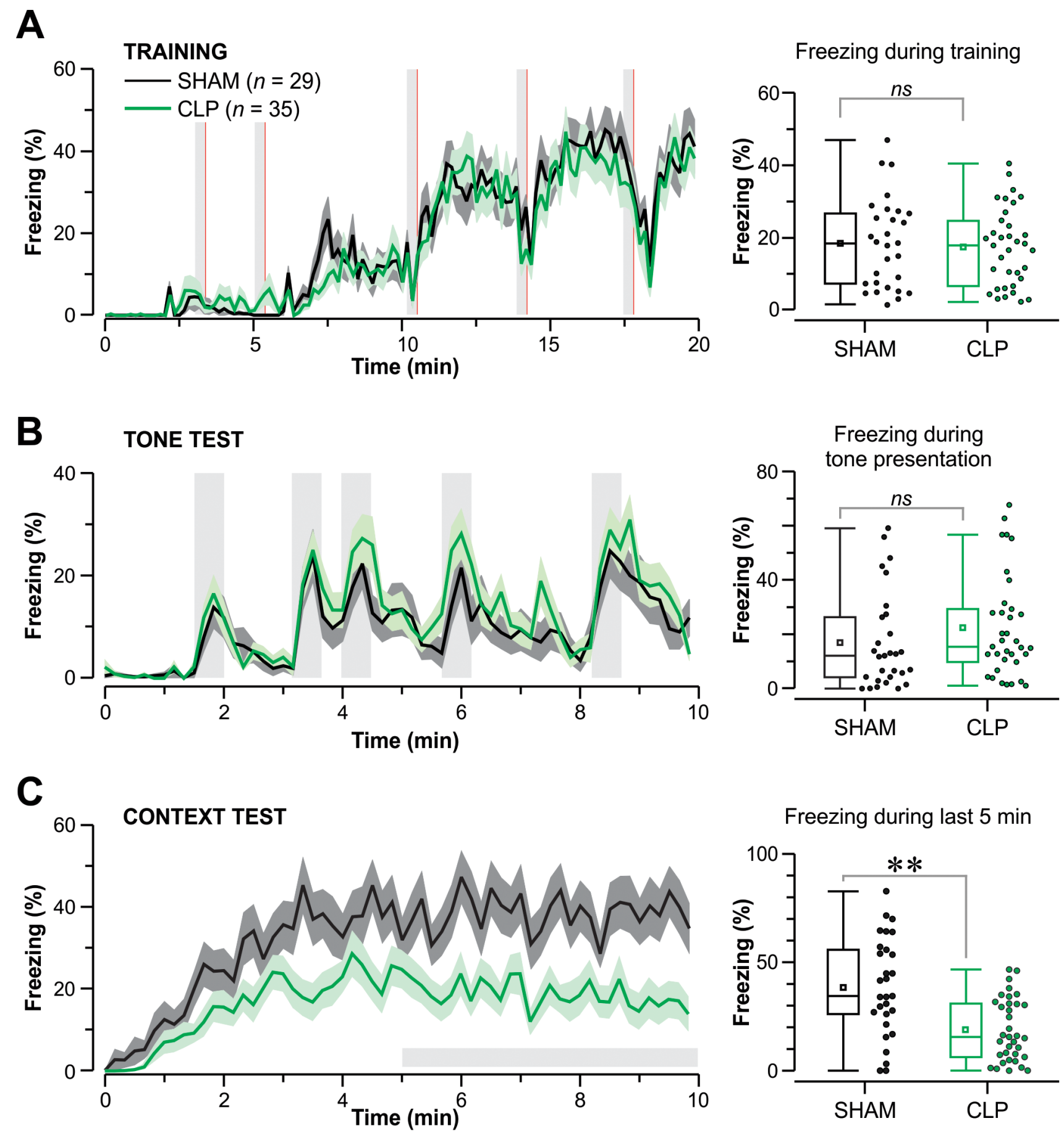

Freezing during last $5 \mathrm{~min}$

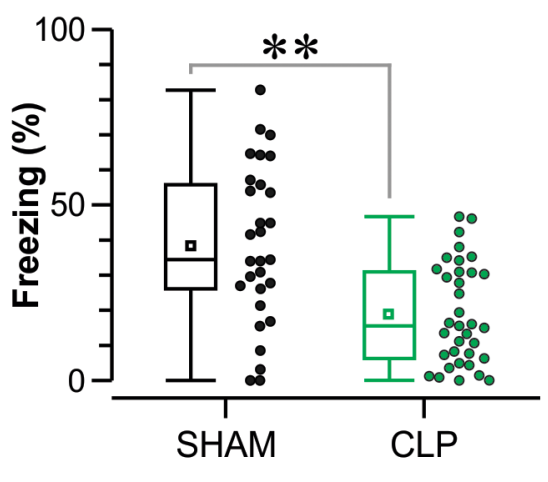

Figure 3. Impaired contextual fear memory in CLP-exposed mice. (A) (Left) The graph shows the progression of freezing (mean \pm SEM) during the training phase. Delivery of the tone CS (vertical light gray bars) coterminates with the foot-shock US (red lines). (Right) The box plot shows the freezing levels during the last $30 \mathrm{~s}$ of training, which are not significantly different (ns) between groups. (B) (Left) The graph shows freezing (mean \pm SEM) during the tone memory test, in which the light gray bars indicate delivery of the tone CS. (Right) The box plot with the freezing levels during the tone presentations, which are similar between groups. (C) (Left) The graph shows freezing (mean \pm SEM) during the context memory test. The gray bar indicates the 5-10 min interval during which the freezing response is calculated. (Right) The box plot shows significantly different freezing during the last $5 \mathrm{~min}\left({ }^{* *} P<0.01\right.$ by $\dagger$ test). 
CONTEXTUAL FEAR MEMORY

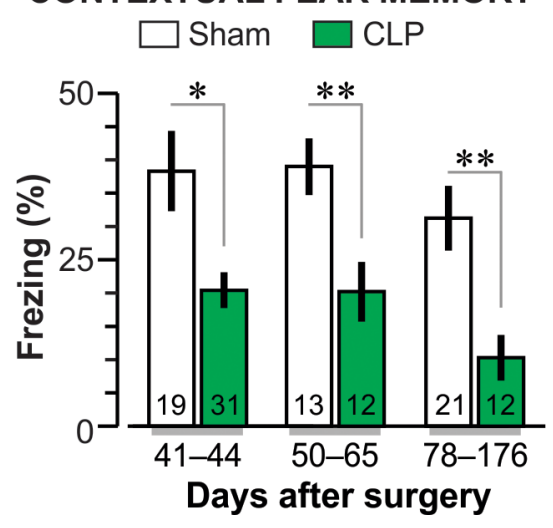

Figure 4. Long-term impairment of contextual fear memory in CLP-exposed mice. Male C57BL/6 mice are subjected to the associative fear conditioning paradigm at different post-surgical time points, and the results are grouped into three intervals. The CLP group shows impairment in the contextual test when compared with the sham group at each time point ( ${ }^{*} P<0.05$; ${ }^{*} P<0.01$ by + test).

\section{DISCUSSION}

This study demonstrates that mice surviving CLP, a model for clinical sepsis, were impaired in contextual fear memory and exhibited significantly fewer dendritic spines in excitatory neurons of the BLA and DG when compared with sham animals. Although there was comparable spine density in the basal dendrites of CA1 pyramidal cells, our published data on the apical dendrites of CA1 pyramidal cells $(10,47)$ revealed marked depletion in the CLP group. In combination, these results suggest a critical involvement of CA1 together with the BLA and DG. Indeed, the contributions of the amygdala and hippocampus in encoding contextual fear memory are well studied; therefore, the specific loss of dendritic spines in the BLA, DG and CA1 strongly suggests a structurefunction nexus for the emotional imbalance occurring in the post-septic brain that is likely to be important as clinical treatment opportunities arise.

Interestingly, CLP-exposed mice were not impaired in the acquisition of fear conditioning, nor were they deficient when tested for auditory fear memory. These results argue that the decreased spine density in BLA neurons do not impede the CLP mice from exhibiting intact cued fear learning and fear tone memory. It is possible that neurons in other subregions of the amygdala, such as the lateral and central amygdala, might be able to compensate for the diminished capacity of BLA neurons to keep tone fear memory intact, a possibility that needs to be investigated. Conversely, it emphasizes the fragility of the more distributed network for contextual fear memory, based on the amygdala and the hippocampus. Surprisingly, the basal dendrites of CA1 neurons do not appear to be affected by sepsis; nevertheless, their intactness does not seem to be sufficient to compensate for the deficient contextual fear memory. Moreover, our results do not rule out the possibility that CLP-exposed mice might exhibit an enhancement in contextual fear extinction, which depends on an even larger network, including the prefrontal cortex together with the hippocampus and amygdala (51).

There is disagreement in the clinical sepsis literature about whether the high prevalence of increased disability after intensive care unit hospitalization is caused by common problems of aging, or whether there is a direct mechanism related to the septic episode (54-56). The results of our study, together with our previous data $(10,47)$ using the CLP model (27), support the idea that sepsis is causally related to impairments in emotional response and spatial memory. In fact, it has been demonstrated that cytokines are neurotoxic agents that mediate brain dysfunction, particularly the cytokine HMGB1 $(10,57)$. While HMGB1 rises late in a septic episode, its potential contribution to chronic neuronal alterations needs further investigation. Nevertheless, sepsis and elevated cytokines might cause behavioral impairments and, despite unclear mechanisms, trigger neuronal loss $(14,17,57)$. There is evidence that immune mediators, and cytokines in particular, alter brain synaptic function $(10,58,59)$, perhaps unsurprisingly, since TNF and IL-1 act as regulators of the density and activity of the key classes of glutamate receptors, AMPARs and NMDARs (60).

The finding that CLP-exposed mice exhibit both defective contextual fear memory and a lower number of dendritic spines in BLA, CA1 and DG aligns well with the established hypothesis on structural plasticity, in which the dynamic turnover of dendritic spines reflects a fundamental substrate for memory. Indeed, recent anatomical studies on amygdalar neurons have shown that spines begin to be added within minutes of the final retention period in a fear conditioning paradigm $(61,62)$. Memory encoding can trigger structural plasticity, but a variety of pharmacological and hormonal challenges can also influence spine dynamics; therefore, it is likely that the elevated cytokine levels associated with a septic event might permanently dampen the plasticity of dendritic spines in BLA, CA1 and DG neurons. It is also noteworthy that experimental and clinical work has demonstrated the association of abnormal dendritic arborization patterns, together with altered dendritic spine density, in various psychiatric and genetic disorders (63-65). Other reports have shown that acquired disorders of the nervous system, including autoimmune disorders, can alter neuronal structural integrity (66-70).

\section{CONCLUSION}

Our study shows that long-term sepsis survivors are impaired in contextual fear memory and exhibit significantly fewer dendritic spines in the excitatory neurons of the amygdala and the hippocampus. Our data are consistent with the possibility that the lowered spine density (observed in BLA excitatory neurons, apical dendrites of CA1 cells and DG granule cells) in CLP-exposed mice might reflect a chronic state, indicative of an altered capacity to replenish the dendritic spines, which in turn might result in a defective fear memory phenotype. Our study strongly suggests that the emotional disturbances experienced by human 
A

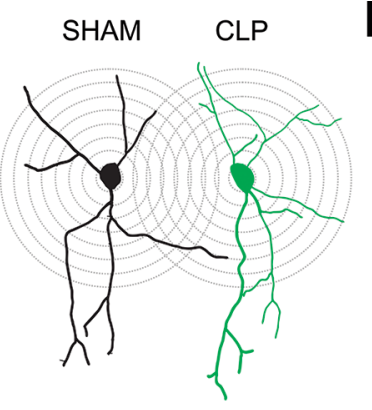

C Primary dendrites

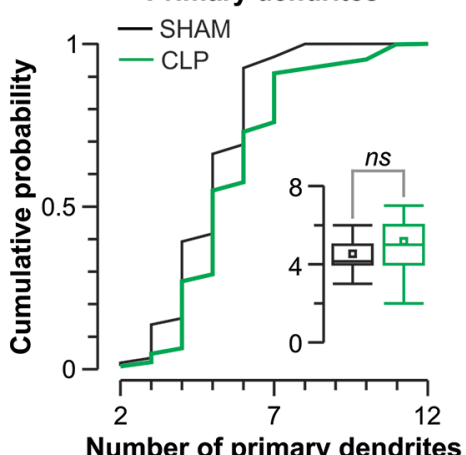

Number of primary dendrites

B
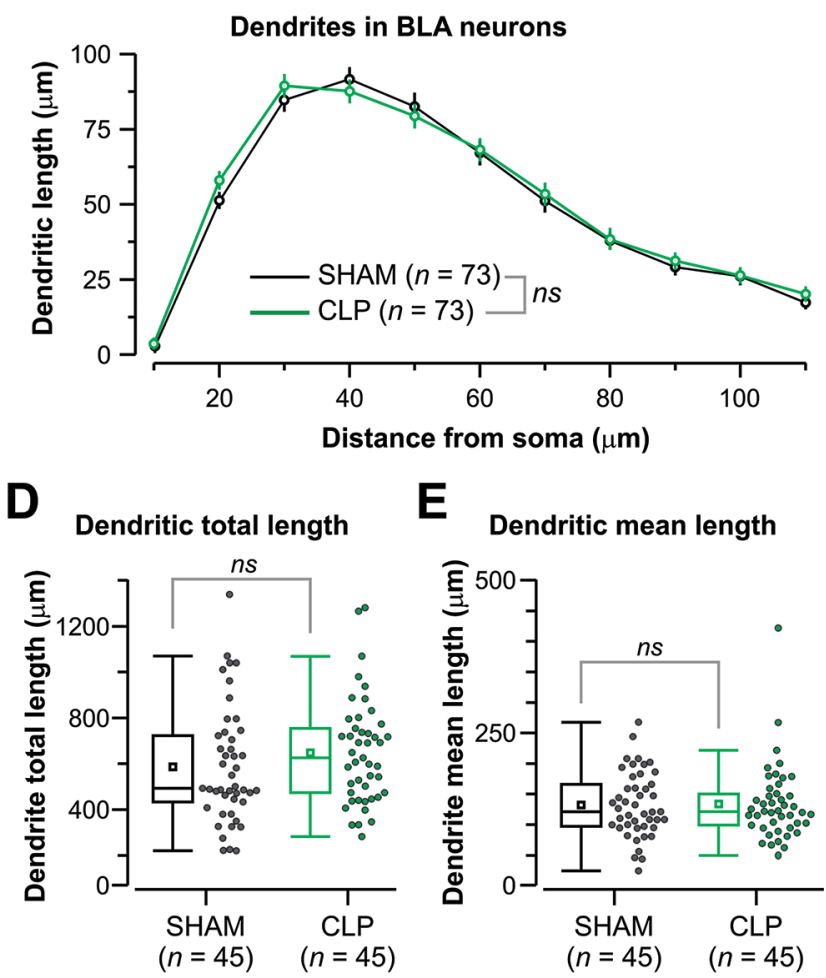

E

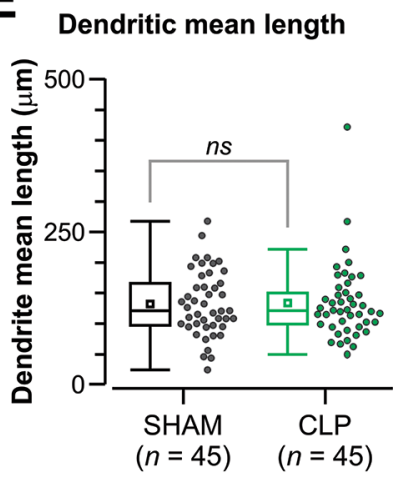

$\mathbf{F}$
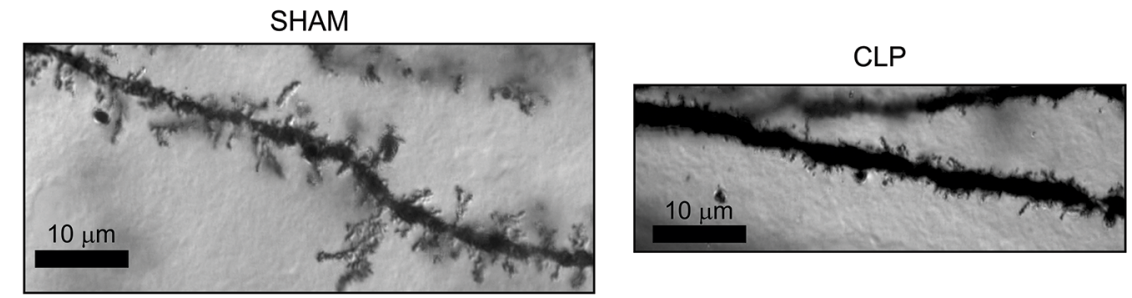

G BLA spines at 8 weeks post-surgery

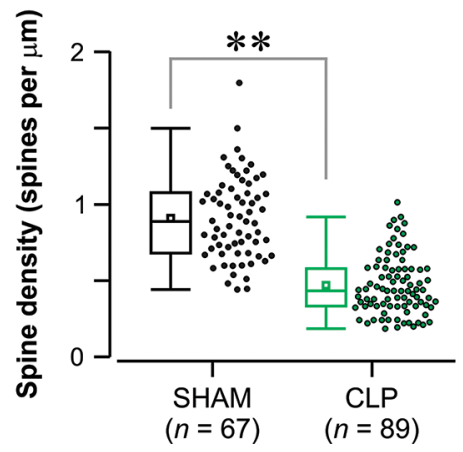

H BLA spines at 24 weeks post-surgery

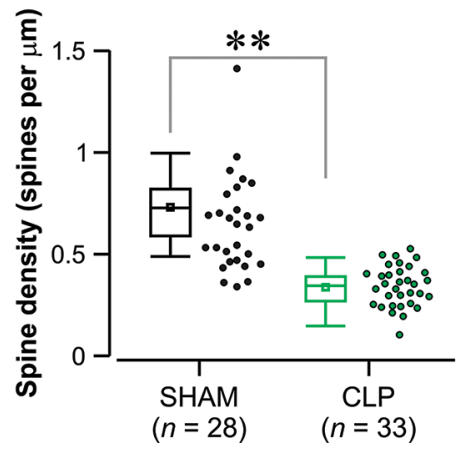

Figure 5. Altered dendritic spine density of BLA neurons in CLP-exposed mice. (A) Traces of representative Golgi-stained BLA neurons from the sham and CLP groups. Concentric rings illustrate the dendritic Sholl analysis, using concentric circles whose diameter expands in $10 \mu \mathrm{m}$ intervals. (B) The graph shows the length of BLA dendrites (obtained from Sholl analysis) versus distance from the cell body. Values are not significantly different between groups ( $n s$, not significant; $P=0.99, D=0.18, Z=042$, Kolmogorov-Smirnov test). (C) The graph shows that the cumulative probability of the distribution of primary dendrites for both groups is comparable (inset shows box plots; $n s, P=0.045$, $t$ test). (D-E) Box plots of dendritic measurements are comparable for total length ( $D, n s, P=0.23, t$ test), and mean length ( $E, n s, P=0.88, t$ test). (F) Micrographs show abundant dendritic spines in the sham dendrite (left) and fewer spines in the CLP dendrite (right). (G-H) Box plots of spine density show that the CLP group has significantly fewer spines than the sham group for the BLA dendrites at $(G) 8$ wks and $(H) 24$ wks post-surgery. Statistics, ${ }^{* *} P<0.01$ by $\dagger$ test. 


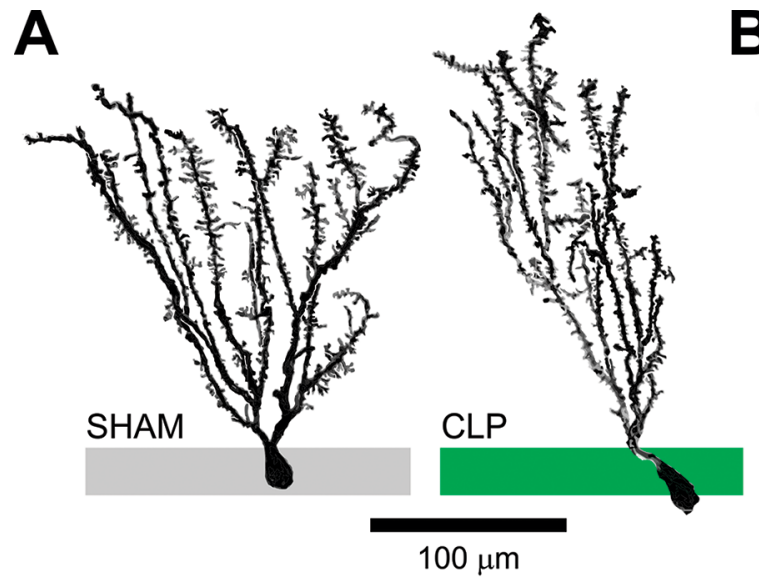

B

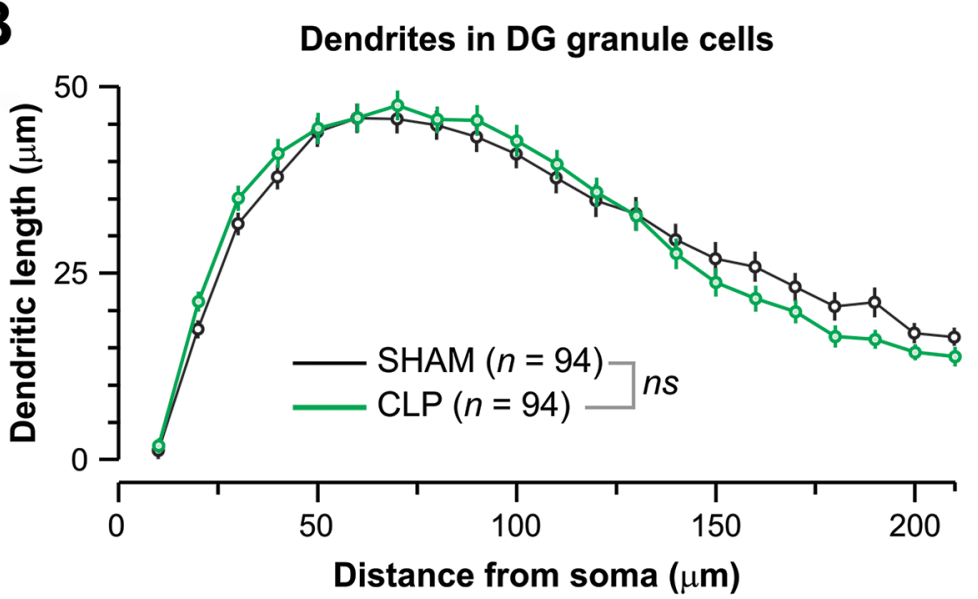

C Primary dendrites

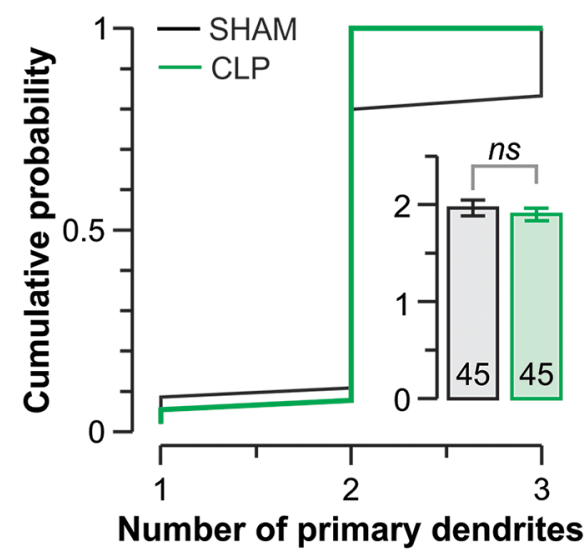

$\mathbf{F}$

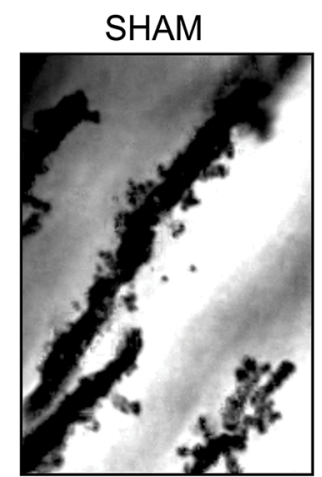

D

Dendritic total length

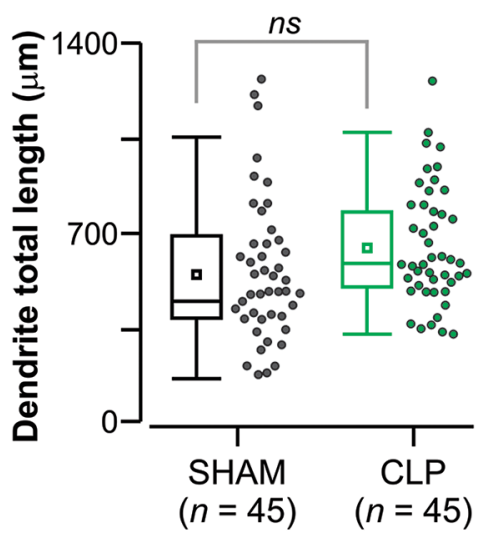

E Dendritic mean length

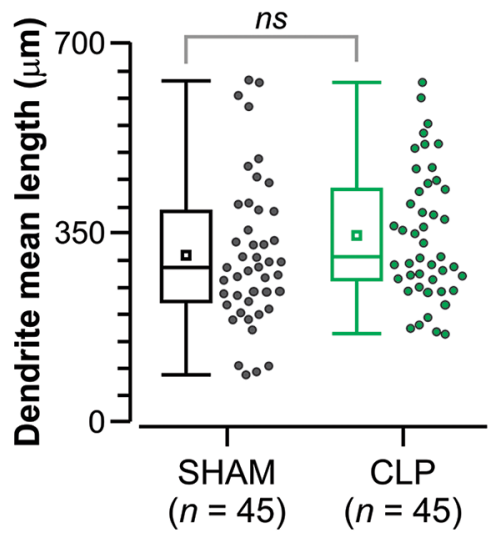

G
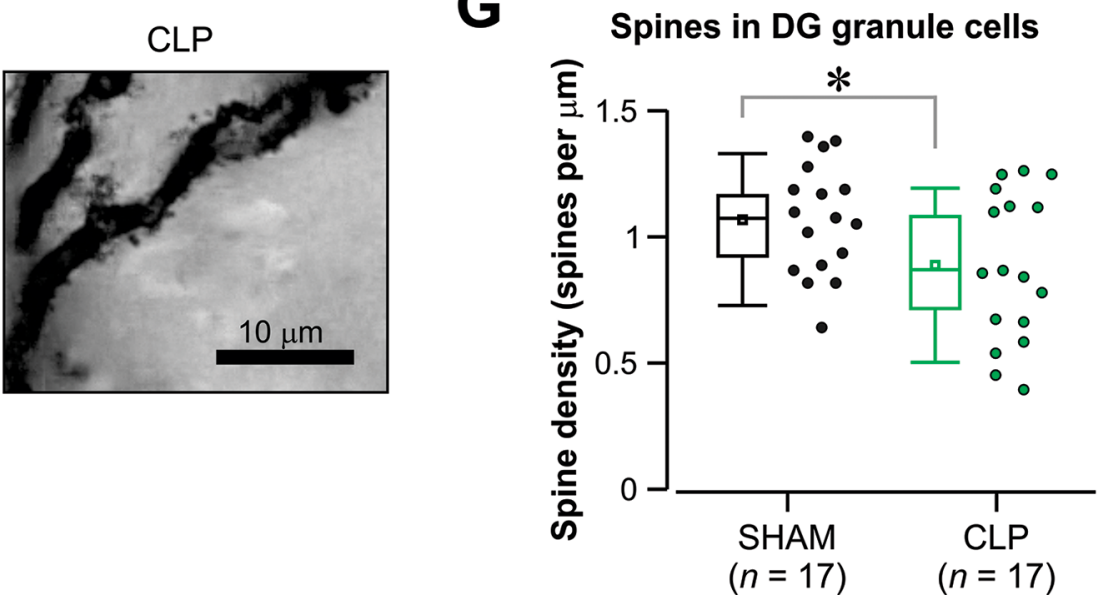

Figure 6. Altered dendritic spine density of DG neurons in CLP-exposed mice. (A) Traces of representative Golgi-stained dentate neurons from the sham and CLP groups. (B) The graph shows the length of DG dendrites (obtained from Sholl analysis) versus distance from the cell body. Values are not significantly different between groups ( $n s$, not significant; $P=0.98, D=0.14, Z=046$, Kolmogorov-Smirnov test). (C) The graph shows that the cumulative probability of the distribution of primary dendrites for both groups is comparable (inset shows box plots; $n s, P=0.47$, $t$ test). ( $D-E)$ Box plots of dendritic measurements are comparable for total length $(D, n s, P=0.09, t$ test) and mean length ( $E, n s, P=0.18$, t test). (F) Micrographs show dendritic spines in DG dendrites. (G) The box plot of spine density shows that the CLP group has significantly fewer spines than the sham group. Statistics, ${ }^{*} P<0.05$ by $\dagger$ test. 

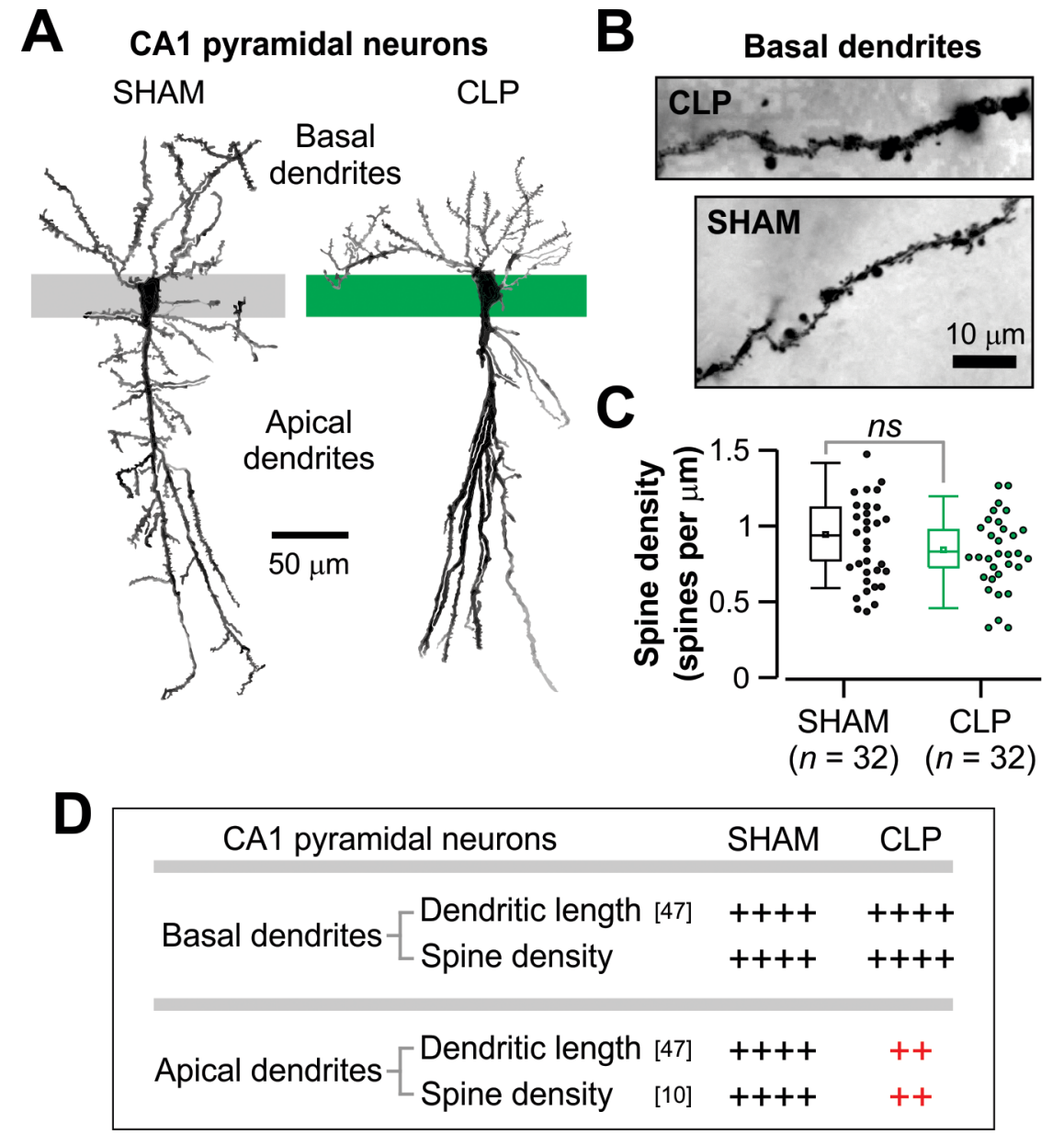

Figure 7. Unaltered basal dendrites in CA1 pyramidal neurons in CLP-exposed mice. (A) Traces of representative Golgi-stained CAI neurons from the sham and CLP groups. (B) Micrographs show dendritic spines in CA1 basal dendrites. (C) The box plot of spine density in CA1 basal dendrites shows that spine counts are similar between groups; ns, nonsignificant by t test. (D) The table summarizes the data for CA 1 pyramidal neurons from this study as well as previous data in references (10) and (47). The basal dendrites are similar between groups in terms of length and spine density, whereas the apical dendrites are disrupted in the CLP group.

patients who have survived septic shock might be the result of subtle damages to the dendritic architecture of neurons of the amygdala and the hippocampus.

\section{ACKNOWLEDGMENTS}

We thank all the members of the Laboratory of Immune and Neural Networks for their help in these studies. This study was funded by National Institutes of Health grants P01-AI073693 (to PH and $\mathrm{BV}$; program director, Betty Diamond) and P01-AI102852 (to PH and BV; program directors, B. Diamond and K. Tracey).

\section{DISCLOSURE}

The authors declare that they have no competing interests as defined by Molecular Medicine, or other interests that might be perceived to influence the results and discussion reported in this paper.

\section{REFERENCES}

1. Hotchkiss RS, Coopersmith CM, McDunn JE, Ferguson TA. (2009) The sepsis seesaw: tilting toward immunosuppression. Nat. Med. 15:496-97.

2. Angus DC et al. (2001) Epidemiology of severe sepsis in the United States: Analysis of incidence, outcome, and associated costs of care. Crit. Care Med. 29:1303-10.

3. Leibovici L et al. (1995) Long-term survival following bacteremia or fungemia. JAMA. 274:807-12.

4. Lillie PJ et al. (2012) Long-term mortality following bloodstream infection. Clin. Microbiol. Infect. 19:955-60.

5. Perl TM, Dvorak L, Hwang T, Wenzel RP. (1995) Long-term survival and function after suspected gram-negative sepsis. JAMA. 274:338-45.

6. Winters BD, et al. (2010) Long-term mortality and quality of life in sepsis: a systematic review. Crit. Care Med. 38:1276-83.

7. Iwashyna TJ, Ely EW, Smith DM, Langa KM. (2010) Long-term cognitive impairment and functional disability among survivors of severe sepsis. JAMA. 304:1787-94.

8. Lazosky A, Young GB, Zirul S, Phillips R. (2010) Quality of life after septic illness. J. Crit. Care. 25:406-12.

9. Semmler A, et al. (2013) Persistent cognitive impairment, hippocampal atrophy and EEG changes in sepsis survivors. J. Neurol. Neurosurg. Psychiatry. 84:62-69.

10. Chavan SS, et al. (2012) HMGB1 mediates cognitive impairment in sepsis survivors. Mol. Med. 18:930-37.

11. Laflamme N, Lacroix S, Rivest S. (1999) An essential role of interleukin- $1 \beta$ in mediating NF- $\kappa B$ activity and COX-2 transcription in cells of the blood-brain barrier in response to a systemic and localized inflammation but not during endotoxemia. J. Neurosci. 19:10923-30.

12. Godbout JP, et al. (2005) Exaggerated neuroinflammation and sickness behavior in aged mice following activation of the peripheral innate immune system. FASEB J. 19:1329-31.

13. Semmler A, Okulla T, Sastre M, Dumitrescu L, Heneka M. (2005) Systemic inflammation induces apoptosis with variable vulnerability of different brain regions. J. Chem. Neuroanat. 30:144-57.

14. Cunningham C, Wilcockson DC, Campion $S$, Lunnon K, Perry VH. (2005) Central and systemic endotoxin challenges exacerbate the local inflammatory response and increase neuronal death during chronic neurodegeneration. J. Neurosci. 25:9275-84.

15. Tuon L, et al. (2007) Imipramine reverses the depressive symptoms in sepsis survivor rats. Intensive Care Med. 33:2165-67.

16. Teeling JL, et al. (2007) Sub-pyrogenic systemic inflammation impacts on brain and behavior, independent of cytokines. Brain Behav. Immun. 21:836-50.

17. Semmler A, et al. (2007) Long-term cognitive impairment, neuronal loss and reduced cortical cholinergic innervation after recovery from sepsis in a rodent model. Exp. Neurol. 204:733-40.

18. Semmler A, et al. (2008) Sepsis causes neuroinflammation and concomitant decrease of cerebral metabolism. J. Neuroinflammation. 5:38.

19. Cunningham C, et al. (2009) Systemic inflammation induces acute behavioral and cognitive changes and accelerates neurodegenerative disease. Biol. Psychiatry. 65:304-12. 
20. Weberpals M, et al. (2009) NOS2 gene deficiency protects from sepsis-induced long-term cognitive deficits. J. Neurosci. 29:14177-84.

21. Cibelli M, et al. (2010) Role of interleukin-1 $\beta$ in postoperative cognitive dysfunction. Ann. Neurol. 68:360-68.

22. Vezzani A, Maroso M, Balosso S, Sanchez MA, Bartfai T. (2011) IL-1 receptor/Toll-like receptor signaling in infection, inflammation, stress and neurodegeneration couples hyperexcitability and seizures. Brain Behav. Immun. 25:1281-89.

23. Jacob A, Brorson JR, Alexander JJ. (2011) Septic encephalopathy: Inflammation in man and mouse. Neurochem. Int. 58:472-76.

24. Mazarati A, Maroso M, Iori V, Vezzani A, Carli M (2011) High-mobility group box-1 impairs memory in mice through both toll-like receptor 4 and receptor for advanced glycation end products. Exp. Neurol. 232:143-48.

25. Terrando N, et al. (2011) Resolving postoperative neuroinflammation and cognitive decline. Ann. Neurol. 70:986-95.

26. Cohen J, et al. (2015) Sepsis: a roadmap for future research. Lancet Infect. Dis. 15:581-614.

27. Dejager L, Pinheiro I, Dejonckheere E, Libert C. (2011) Cecal ligation and puncture: the gold standard model for polymicrobial sepsis? Trends Microbiol. 19:198-208.

28. Bone RC. (1991) Gram-negative sepsis. Background, clinical features, and intervention. Chest. 100:802-08.

29. Boomer JS, et al. (2011) Immunosuppression in patients who die of sepsis and multiple organ failure. JAMA. 306:2594-605.

30. Brunkhorst FM, et al. (2012) Effect of empirical treatment with moxifloxacin and meropenem vs meropenem on sepsis-related organ dysfunction in patients with severe sepsis: a randomized trial. JAMA. 307:2390-99.

31. Dellinger RP, et al. (2008) Surviving Sepsis Campaign: international guidelines for management of severe sepsis and septic shock: 2008. Crit. Care Med. 36:296-327.

32. Kreger BE, Craven DE, McCabe WR. (1980) Gram-negative bacteremia. IV. Re-evaluation of clinical features and treatment in 612 patients. Am. J. Med. 68:344-55.

33. Martin GS, Mannino DM, Eaton S, Moss M. (2003) The epidemiology of sepsis in the United States from 1979 through 2000. N. Engl. J. Med. 348:1546-54.

34. Barichello T, et al. (2005) Long-term cognitive impairment in sepsis survivors. Crit. Care Med. 33:221-23.

35. Barichello T, et al. (2007) Antioxidant treatment prevented late memory impairment in an animal model of sepsis. Crit. Care Med. 35:2186-90.

36. Tuon L, et al. (2008) Memory-enhancing treatments reverse the impairment of inhibitory avoidance retention in sepsis-surviving rats. Crit. Care. 12:R133.

37. Tuon L, et al. (2008) Time-dependent behavioral recovery after sepsis in rats. Intensive Care Med. 34:1724-31.

38. Calsavara AC, et al. (2013) Late anxiety-like behavior and neuroinflammation in mice subjected to sublethal polymicrobial sepsis. Neurotox. Res. 24:103-08.

39. Michels M, et al. (2015) CD40-CD40 ligand pathway is a major component of acute neuroinflammation and contributes to long-term cognitive dysfunction after sepsis. Mol. Med. 21:219-26.

40. Moraes CA, et al. (2015) Activated microgliainduced deficits in excitatory synapses through IL-1 $\beta$ : Implications for cognitive impairment in sepsis. Mol. Neurobiol. 52:653-63.

41. Terrando N, et al. (2010) Tumor necrosis factoralpha triggers a cytokine cascade yielding postoperative cognitive decline. Proc. Natl. Acad. Sci. USA. 107:20518-22.

42. Gao R, et al. (2015) Systemic lipopolysaccharide administration-induced cognitive impairments are reversed by erythropoietin treatment in mice. Inflammation. 38:1949-58.

43. Wu J, et al. (2015) Mitochondria-targeted peptide reverses mitochondrial dysfunction and cognitive deficits in sepsis-associated encephalopathy. Mol. Neurobiol. 52:783-91.

44. Li R, et al. (2015) Low molecular weight heparin prevents lipopolysaccharide-induced hippocampus-dependent cognitive impairments in mice. Int. J. Clin. Exp. Pathol. 8:8881-91.

45. Gao R, Kan MQ, Wang SG, Yang RH, Zhang SG. (2016) Disrupted tryptophan metabolism induced cognitive impairment in a mouse model of sepsis-associated encephalopathy. Inflammation. 39:550-60.

46. Singer BH, et al. (2016) Cecal ligation and puncture results in long-term central nervous system myeloid inflammation. PLoS One. 11:e0149136.

47. Volpe BT, Berlin RA, Frankfurt M. (2015) The brain at risk: the sepsis syndrome and lessons from preclinical experiments. Immunol. Res. 63:70-74.

48. LeDoux JE. (2000) Emotion circuits in the brain. Annu. Rev. Neurosci. 23:155-84.

49. Fanselow MS, Poulos AM. (2005) The neuroscience of mammalian associative learning. Annu. Rev. Psychol. 56:207-34.

50. Huerta PT, Kowal C, DeGiorgio LA, Volpe BT, Diamond B. (2006) Immunity and behavior: antibodies alter emotion. Proc. Natl. Acad. Sci. USA. 103:678-83.

51. Tovote P, Fadok JP, Luthi A. (2015) Neuronal circuits for fear and anxiety. Nat. Rev. Neurosci. 16:317-31.

52. H. Yang H, et al. (2004) Reversing established sepsis with antagonists of endogenous highmobility group box 1. Proc. Natl. Acad. Sci. USA. 101:296-301.

53. Rogers DC, et al. (1997) Behavioral and functional analysis of mouse phenotype: SHIRPA, a proposed protocol for comprehensive phenotype assessment. Mamm. Genome. 8:711-13.

54. Cigolle CT, et al. (2011) Geriatric conditions develop in middle-aged adults with diabetes. J. Gen. Intern. Med. 26:272-79.

55. Iwashyna TJ, Netzer G, Langa KM, Cigolle C. (2012) Spurious inferences about long-term outcomes: the case of severe sepsis and geriatric conditions. Am. J. Respir. Crit. Care Med. 185:835-41.

56. Rubenfeld GD. (2012) Does the hospital make you older faster? Am. J. Respir. Crit. Care Med. 185:796-68.

57. Wang H, Yang H, Czura CJ, Sama AE, Tracey KJ. (2001) HMGB1 as a late mediator of lethal systemic inflammation. Am. J. Respir. Crit. Care Med. 164:1768-73

58. McAfoose J, Baune BT. (2009) Evidence for a cytokine model of cognitive function. Neurosci. Biobehav. Rev. 33:355-66.

59. McCoy M, Tansey M. (2008) TNF signaling inhibition in the CNS: implications for normal brain function and neurodegenerative disease. J. Neuroinflammation. 5:45.

60. Stellwagen D, Malenka RC. (2006) Synaptic scaling mediated by glial TNF-alpha. Nature. 440:1054-59.

61. Eilam-Stock T, Serrano P, Frankfurt M, Luine V. (2012) Bisphenol-A impairs memory and reduces dendritic spine density in adult male rats. Behav. Neurosci. 126:175-85

62. Heinrichs SC, et al. (2013) Dendritic structural plasticity in the basolateral amygdala after fear conditioning and its extinction in mice. Behav. Brain Res. 248:80-84.

63. Goellner B, Aberle H. (2012) The synaptic cytoskeleton in development and disease. Dev. Neurobiol. 72:111-25.

64. Koleske AJ. (2013) Molecular mechanisms of dendrite stability. Nat. Rev. Neurosci. 14:536-50.

65. Kulkarni VA, Firestein BL. (2012) The dendritic tree and brain disorders. Mol. Cell. Neurosci. 50:10-20.

66. Fiala JC, Spacek J, Harris KM. (2002) Dendritic spine pathology: cause or consequence of neurological disorders? Brain Res. Brain Res. Rev. 39:29-54.

67. Lin YC, Koleske AJ. (2010) Mechanisms of synapse and dendrite maintenance and their disruption in psychiatric and neurodegenerative disorders. Annu. Rev. Neurosci. 33:349-78.

68. Gillingwater TH, Wishart TM. (2013) Mechanisms underlying synaptic vulnerability and degeneration in neurodegenerative disease. Neuropathol. Appl. Neurobiol. 39:320-34.

69. Chang EH, et al. (2015) Selective impairment of spatial cognition caused by autoantibodies to the N-methyl-D-aspartate receptor. EBioMedicine. 2:755-64.

70. Frauenknecht K, et al. (2015) Mice with experimental antiphospholipid syndrome display hippocampal dysfunction and a reduction of dendritic complexity in hippocampal CA1 neurones. Neuropathol. Appl. Neurobiol. 41:657-71.

Cite this article as: Huerta PT, et al. (2016) Preclinical models of overwhelming sepsis implicate the neural system that encodes contextual fear memory. Mol. Med. 22:789-99. 